\title{
ON DYNAMICAL SYSTEMS WITH THE SPECIFICATION PROPERTY
}

\author{
BY
}

\section{KARL SIGMUND}

ABSTRACT. A continuous transformation $T$ of a compact metric space $X$ satisfies the specification property if one can approximate distinct pieces of orbits by single periodic orbits with a certain uniformity. There are many examples of such transformations which have recently been studied in ergodic theory and statistical mechanics. This paper investigates the relation between $T$ invariant measures and the frequencies of $T$-orbits. In particular, it is shown that every invariant measure (and even every closed connected subset of such measures) has generic points, but that the set of all generic points is of first category in $X$. This generalizes number theoretic results conceming decimal expansions and normal numbers.

1. Introduction. A number $x \in[0,1)$ is said to be normal to the base $s$ ( $s$ positive integer) if in the s-adic expansion $0 . x_{1} x_{2} \cdots$ of $x$ every possible $k$ block occurs with frequency $s^{-k}(k>1,2, \ldots)$. This is equivalent to the condition that the sequence $s^{n} x(\bmod 1)$ is uniformly distributed in $[0,1)$, i.e. that for every continuous real-valued function $f$ with period 1 , one has

$$
\lim _{N \rightarrow \infty} \frac{1}{N} \sum_{n=0}^{N-1} f\left(s^{n} x\right)=\int_{0}^{1} f d m
$$

where $m$ denotes Lebesgue measure.

Let $T_{s}$ denote the map $x \mapsto s x$ (mod 1$)$ from $[0,1)$ onto itself, and write $\mu\left(x, T_{s} ; N\right)$ for the measure with mass $1 / N$ at the points $x, s x, \cdots$, $s^{N-1} x(\bmod 1)$. It is easy to see that the set $V^{I_{s}}(x)$ of accumulation points of $\mu\left(x, T_{s} ; N\right)$ is a nonempty closed connected subset of the space of $T_{s}$-invariant measures on $[0,1)$ provided with the weak topology for measures. If this subset consists of $m$ alone, then $x$ is normal to the base $s$. Almost all $x$ are normal. But it has been shown in a series of papers on nonnormal numbers that for every closed connected set $V \neq \varnothing$ of $T_{s}$-invariant measures on $[0,1)$ there is an $x \epsilon$ $[0,1)$ such that $V^{T_{s}}(x)=V$ (see [6]). This corresponds to a statement about the frequencies of $k$-blocks in the $s$-adic expansion of $x$, which is based on the

Received by the editors April 2, 1973.

AMS (MOS) subject classifications (1970). Primary 28A65; Secondary 54H20, $10 \mathrm{~K} 10$.

Key words and phrases. Ergodic measures, generic points, quasiregular points, normal numbers. 
fact that there is no obstacle to the construction of $s$-adic expansions by concatenating arbitrary sequences of $k$-blocks. This means that one can specify the $T_{s}$-orbit of some $x \in[0,1)$ such that one gets just the "right" frequencies, by concatenating, so to speak, arbitrary sequences of pieces of $T_{s}$-orbits.

A specification property which allows one to construct in a similar way orbits for much larger classes of transformations has been first considered by Bowen in [2], and was used later in [4], [5], [16], [18], [19] and [20]. In this paper, we prove for such transformations theorems concerning invariant measures and generic points. They can be viewed as extensions of some results obtained for $s$ adic expansions in [8], [23] and [6].

2. Definitions and elementary properties. Let $(X, T)$ denote the dynamical system consisting of the compact metric space $X$ and the continuous map $T$ from $X$ onto itself. Let $d$ denote the distance in $X$. We shall tacitly assume $T$ to be a homeomorphism whenever this is necessary-i.e. whenever negative powers of $T$ are considered. By a string $A=[a, b]$ we mean a finite set of consecutive integers $\{a, a+1, \ldots, b\}$. By a piece of orbit we mean a set $\left\{T^{j} x: x \in X, j \in A\right\}$.

Definition. $(X, T)$ is said to have the specification property $((X, T) \in \mathrm{SPEC})$ if for every $\epsilon>0$ there is an integer $M(\epsilon)$ such that for any choice of points $x_{1}$, $x_{2} \in X$ and strings $A_{1}=\left[a_{1}, b_{1}\right], A_{2}=\left[a_{2}, b_{2}\right]$ with $a_{2}-b_{1}>M(\epsilon)$, and any integer $p>b_{2}-a_{1}+M(\epsilon)$, there exists a periodic point $x \in X$ with period $p$ such that

$$
\begin{array}{ll}
d\left(T^{j} x_{0} T^{j} x_{1}\right)<\epsilon & \text { for } j \in A_{1}, \\
d\left(T^{j} x, T^{j} x_{2}\right)<\epsilon & \text { for } j \in A_{2} .
\end{array}
$$

(This definition does not depend on the choice of the metric $d_{\text {.) }}$ )

The motivation for this definition comes from the wish to approximate simultaneously, up to $\epsilon$, two finite pieces of orbit $\left\{T^{j} x_{1}: j \in A_{1}\right\}$ and $\left\{T^{j} x_{2}: j \in A_{2}\right\}$ by one periodic orbit. Obviously $A_{1}$ and $A_{2}$ have to be disjoint: one would like the gap between $A_{1}$ and $A_{2}$, which corresponds to the time for "switching over" from one piece of orbit to the other, to be as small as possible. Also, one would like the length of the periodic orbit to be as small as possible: this means that the time for "switching back" from the second piece of orbit to the first one should also be small. If $(X, T)$ satisfies the specification property, such a simultaneous approximation is possible, provided that the times for switching from one piece of orbit to the other are larger than $M(\epsilon)$. This number depends neither on the approximated orbits, i.e. on the choice of $x_{1}$ and $x_{2}$, nor on the cardinality of $A_{1}$ and $A_{2}$, i.e. on the length of time that the periodic orbit is specified up to $\epsilon$. 
This seems a very strong condition, but there are many examples of dynamical systems satisfying it:

(A) Let $M$ be a compact metric space, and $X=\Pi_{-\infty}^{+\infty} M$ the product space with product topology. An element $x$ of $X$ is a bilateral sequence $\left(x_{n}\right)_{n \in Z}$ of elements of $M$. Let $T$ be the shift $(T x)_{n}=x_{n+1}, n \in Z$. Obviously $(X, T)$ has the specification property.

(B) If $M$ is a finite discrete space, one obtains by (A) the shift on a finite alphabet; this transformation can also be "represented" as transformation on the unit interval $l=[0,1)$ by $x \mapsto s x(\bmod 1)$ for $s=$ card $M$. This is an example of an expanding differentiable map (see [17]). Such maps have the specification property, because they can be displayed as factors of the shift on a finite alphabet (see [11] and Proposition 1(b)).

(C) It is an easy exercise to characterize the class of subshifts of the shift on a finite alphabet which have the specification property. In particular, subshifts of finite type and sofic subshifts (see [1] and [25]) belong to this class, provided they satisfy some mild mixing condition.

(D) In [2] Bowen proves that if $T$ is the restriction of an Axiom A diffeomorphism to its nonwandering set $\Omega$, then $\Omega$ decomposes into finitely many disjoint pieces $\Omega_{i}$ invariant under some power $T^{k}$ and such that $T^{k} \mid \Omega_{i}$ has the specification property.

(E) In particular, if $X$ is the $n$-dimensional torus and $T$ an automorphism of $X$ induced by a matrix from $S L(n, Z)$ whose eigenvalues are off the unit circle, then $(X, T)$ has the specification property. It would be interesting to see whether this is still true when one only assumes that the eigenvalues are not roots of unity, i.e. that $T$ is ergodic with respect to Haar measure.

(F) It is easy to define the analogue of the specification property for oneparameter flows $\left\{T_{t}: X \rightarrow X\right\}_{t \in R^{*}}$ In [4, Theorem 3.8] Bowen shows that $C$ dense Axiom $A$ flows have this property. In particular, geodesic flows on manifolds of negative curvature belong to this class. It is easy to see that Theorems $1,2,3$ and 5 below, as well as simple modifications of Theorems 4 and 6, are valid for flows with the specification property.

(G) In [16] Ruelle defines and studies specification for $Z^{\nu}$-actions.

Actually the specification properties given by Bowen and Ruelle are slightly different from the one given here.

Bowen's definition in [2] is: For every $\epsilon>0$ there is a $M(\epsilon)$ such that if $x_{1}, \cdots, x_{k}$ are points in $X$ and $A_{i}=\left[a_{i}, b_{i}\right](i=1,2, \ldots, k)$ are strings with $a_{i}-b_{i-1}>M(\epsilon)$ for $i=2,3, \cdots, k$ and if $p>b_{k}-a_{1}+M(\epsilon)$, then there exists a periodic $x \in X$ with period $p$ such that

$$
d\left(T^{j} x_{,} T^{j} x_{i}\right)<\epsilon \text { for } j \in A_{i}, i=1, \ldots, k_{\text {o }}
$$


Thus the periodic orbit here approximates simultaneously $k$ pieces of orbit, and the length of the gaps between the $A_{i}$ 's does not depend on $k$.

In [16] Ruelle calls this the strong specification property. He also has a weak specification: it is the same as Bowen's, except that $x$ is not required to be periodic (hence no condition on $p$ ). The definition used here is in between strong and weak specification.

The class SPEC of systems with the specification property is natural in the sense that it is closed under factors and products. Recall that a factor (or homomorphic image $)$ of $(X, T)$ is a system $(Y, S)$ such that there exists a continuous map $\phi$ from $X$ onto $Y$ with $S \circ \phi=\phi \circ T$. The product of two systems $(X, T)$ and $(Y, S)$ is the system defined on the space $X \times Y$ by the map $T \times S:(x, y) \mapsto$ $(T x, S y)$.

Proposition 1. (a) $(X, T) \in$ SPEC iff $\left(X, T^{k}\right) \in$ SPEC for some $k \neq 0$;

(b) the factor of a system in SPEC is in SPEC;

(c) the product of two systems in SPEC is in SPEC.

We leave the proof as an easy exercise.

Proposition 2. If $(X, T)$ satisfies the specification property, then

(a) the periodic points are dense;

(b) $(X, T)$ is topologically mixing.

This is also easy to check. (Recall that $(X, T)$ is topologically mixing if for all open $U, V \subset X$, there is an $N>0$ such that $T^{-n} U \cap V \neq \varnothing$ for all $n>N$.) It follows that the powers of $T$ are topologically transitive, i.e. that for every $k \neq 0$ there is an $x$ such that $\left\{T^{j k} x\right\}_{j}$ is dense in $X$. In particular, $(X, T)$ is an $F_{\text {flow }}$ and therefore disjoint from all minimal flows (see [10]). Remark also that if $(X, T) \in$ SPEC and $X$ has less than $c$ points, then $X$ reduces to one point.

The following lemma is a technicality useful for the study of averaging behaviour of orbits of $(X, T)$.

Orbit specification lemma. Let $(X, T)$ be a system with the specification property. Let $\epsilon_{n}(n \in N$ or $n \in Z)$ be a given sequence of positive numbers. Then there exists a sequence $M_{k}(k \in N)$ of positive integers sucb that for any increasing sequence of disjoint strings $A_{n}=\left[a_{n}, b_{n}\right]$ witb the property that $a_{n+1}$ $-b_{n}>M_{|n|}$, and for any sequence $x_{n} \in X$, there exists a $z \in X$ sucb that

$$
d\left(T^{j} z, T^{j} x_{n}\right) \leq \epsilon_{n} \text { for } j \in A_{n} \text { all } n \text {. }
$$

Proof. We consider the case of bilateral sequences, $n=0, \pm 1, \pm 2, \ldots$. For $k \geq 0$ write $\eta_{k}=\min |n|<k \epsilon_{n}$ and set $M_{k}=M\left(\eta_{k+1} / 2^{k+3}\right)$. Let $A_{n}, n \in Z$, be a sequence of disjoint strings such that $a_{n+1}-a_{n}>M_{k}$ for $|n|=k$. 
Define a sequence of strings $B_{n}$ as follows: $B_{0}=A ; B_{k}=\left[a_{-(k-1)}, b_{k}\right]$ and $B_{-k}=\left[a_{-k}, b_{k}\right]$ for $k>0$. Define a sequence $z_{n} \in X$ by induction: $z_{0}=$ $x_{0}$; and, if $z_{n}$ has been defined for $|n|<k$, let $z_{k}$ be such that

$$
\begin{gathered}
d\left(T^{j} z_{k}, T^{j} z_{-(k-1)}\right)<\eta_{k} / 2^{k+2} \text { for } j \in B_{-(k-1)}, \\
d\left(T^{j} z_{k}, T^{j} x_{k}\right)<\eta_{k} / 2^{k+2} \text { for } j \in A_{k}
\end{gathered}
$$

and $z_{-k}$ such that

$$
\begin{gathered}
d\left(T^{j} z_{-k}, T^{j} z_{k}\right)<\eta_{k} / 2^{k+2} \text { for } j \in B_{k^{\prime}} \\
d\left(T^{j} z_{-k}, T^{j} x_{-k}\right)<\eta_{k} / 2^{k+2} \text { for } j \in A_{-k^{\bullet}}
\end{gathered}
$$

Such $z_{k}$ (resp. $z_{-k}$ ) exist by the specification property, since $a_{k}-b_{k-1}>M_{k-1}$ (resp. $a_{-(k-1)}-b_{-k}>M_{k}>M_{k-1}$ ). One can see by induction that if $n, m \in Z,|n|>$ $|m|$, then

$$
d\left(T^{j} z_{m}, T^{j} x_{n}\right)<\epsilon_{n} / 2^{n+1} \text { for } j \in A_{n} \text {. }
$$

Let $z$ be the limit of the Cauchy sequence $z_{k}, k>0$. We claim that (1) holds. Indeed, choose $k$ so large that $k>|n|$ and such that

$$
d\left(T^{j} z, T^{j} z_{k}\right)<\epsilon_{n} / 2 \text { for } j \in B_{n} \text {. }
$$

Then for $j \in A_{n}$ one has by (2) and (3)

$$
d\left(T^{j} z, T^{j} x_{n}\right) \leq \epsilon_{n} / 2+\epsilon_{n} / 2^{n+1}<\epsilon_{n}
$$

This lemma allows one to build orbits almost at will. Remark that the length of the strings $A_{n}$ along which $z$ is specified can be arbitrarily large. In a situation like (1) we say that $z$ is specified along the string $A_{n}$ by the orbit of $x_{n}$ up to $\epsilon_{n} \cdot$

In a similar way one can show that for any $\epsilon>0$ and any integer $k>1$, there is a positive integer $M_{\epsilon, k}$ such that for any $x_{i} \in X$, and any set of strings $A_{i}=$ $\left[a_{i}, b_{i}\right]$, with $a_{i+1}-b_{i}>M_{\epsilon_{0} k}$, for $i=1, \ldots, k$ and any integer $p>b_{k}-a_{1}+$ $M_{\epsilon, k}$, there is a periodic point $z \in X$ with period $p$ such that $d\left(T^{j} z, T^{j} x_{i}\right)<\epsilon$ for $j \in A_{i}, i=1, \ldots, k$. From this follows

Proposition 3. If $(X, T)$ is a nontrivial system with the specification property, then the topological entropy of $(X, T)$ is larger than 0.

This proposition has been proved, for one parameter flows, in [4, Theorem 4.12]. The proof is just the same in the discrete case. For a definition of topological entropy, see [3]

3. Generic points and the space of invariant measures. Let $T$ be a continuous map from $X$ onto itself. Let $\bar{M}$ denote the set of all normalized Borel 
measures on $X$, with the weak topology for measures (see [15]). Then $\mu_{n} \rightarrow \mu$ iff

$$
\int f d \mu_{n} \rightarrow \int f d \mu
$$

for all $f \in C(X) . \bar{M}$ is a compact metrizable space $[15$, p. 40]. Let $M(T)$ be the subset of $\bar{M}$ consisting of the $T$-invariant measures: $\mu \in M(T)$ iff

$$
\int(f \circ T) d \mu=\int f d \mu \text { for all } f \in C(X)
$$

It is well known that $M(T)$ is a nonempty convex subset of $M$. The extremal points of $M(T)$ are just the ergodic measures on $X$, i.e. those $\mu \in M(T)$ which do not assign positive measure to two disjoint $T$-invariant Borel sets in $X$.

For $x \in X$ and $N \geq 0$ let $\mu(x, T ; N)$ denote the measure given by the map

$$
f \mapsto \frac{1}{N} \sum_{j=0}^{N-1} f\left(T^{j} x\right), \quad f \in C(X) .
$$

Let $V^{T}(x)$ denote the set of limit points of the sequence $\mu(x, T ; N)$. Since $\bar{M}$ is compact, $V^{T}(x) \neq \varnothing$. It is easy to see that $V^{T}(x)$ is a closed connected subset of $M(T)$. If $V^{T}(x)$ consists of just one point $\mu \in M(T)$, then one says that $x$ is a generic point for $\mu$ with respect to $T$, and writes $\mu=\mu(x, T)$. We shall show in Theorem 4 that if $(X, T)$ has the specification property, then every nonempty closed connected subset of $M(T)$ is such a $V^{T}(x)$. This need not be true if the specification property does not hold. It can happen that there are $\mu \epsilon$ $M(T)$ such that there exists no generic point for $\mu$ (see [24]). If $\mu$ is ergodic, however, then $V^{T}(x)=\{\mu\}$ for $\mu$-almost all $x \in X$ (this follows from Birkhoff's ergodic theorem).

If $x$ is periodic with minimal period $p$, then $x$ is generic for the measure $\mu(x, T)=\mu(x, T ; p)$. Such measures are called CO-measures (because $x$ has a closed orbit). Obviously a CO-measure is ergodic.

Theorem 1. Let $(X, T)$ be a dynamical system with the specification property. Then the set of CO-measures is dense in $M(T)$. More precisely for any two open sets $U \subset M(T), V \subset X$ and any $N>0$, there exists a periodic point $x \in$ $V$ with period $p \geq N$ such that $\mu(x, T) \in U$.

The proof is very similar to the proof of Lemma 1 in [18] or of the corresponding statement for $Z^{\nu}$-actions in [16]. (It was proved there for the "strong specification property", but one does not need this extra strength.) One can construct subshifts showing that the "converse" is not true: there exist dynamical systems which are topologically mixing, have periodic points dense in $X$ and $\mathrm{CO}$ measures dense in $M(T)$, but which do not satisfy the specification property. 
As an immediate corollary of Theorem 1 and the fact that the set of extremal points of a convex set is a $G_{\delta}$, one obtains that the set of ergodic measures is the complement of a set of first category in $M(T)$.

Actually, one can show slightly more. Let $E_{l}$ denote the set of those $\mu \epsilon$ $M(T) \subset M\left(T^{l}\right)$ which are ergodic with respect to $T^{l}(l=1,2, \ldots)$. If $\mu \epsilon$ $\bigcap_{l=1}^{\infty} E_{l}$, then $\mu$ is said to be totally ergodic. If $x$ has prime period $p>l$, then $\mu(x, T) \in E_{l}$. As a consequence of Theorem 1 one obtains therefore

Theorem 2. If $(X, T)$ is a system with the specification property, then the set of totally ergodic measures is the complement of a set of first category in $M(T)$.

Just as in [18], one obtains furthermore

Theorem 3. If $(X, T)$ bas the specification property, then the set of nonatomic measures, the set of measures positive on all open sets, and the set of measures vanishing on all proper closed invariant subsets of $X$ are complements of sets of first category in $M(T)$. The set of strongly mixing measures is a set of first category in $M(T)$.

Recall that a $\mu \in M(T)$ is said to be strongly mixing if $\mu\left(U \cap T^{-n} V\right) \rightarrow$ $\mu(U) \mu(V)$ for all Borel sets $U, V \subset X$. A measure $\mu \in M(T)$ is said to be weakly mixing if for all such $U, V$,

$$
\lim _{N \rightarrow \infty} \frac{1}{N} \sum_{j=0}^{N-1}\left|\mu\left(U \cap T^{-i} V\right)-\mu(U) \mu(V)\right|=0 .
$$

Conjecture. If $(X, T) \in \mathrm{SPEC}$, then the set of weakly mixing measures and the set of measures with zero entropy are complements of sets of first category.

The first part of the conjecture has been verified in special cases in [14] and [20]. The second part has been proved under the further assumption of expansiveness by Ruelle in [16].

Theorem 4. Let $(X, T)$ be a system with the specification property, and $V \subset M(T)$ a nonempty closed connected set. Then there exists an $x \in X$ with $V^{T^{l}}(x)=V$ for $l=1,2, \ldots$.

Proof. By $\left[15\right.$, Theorem 6.6] there is a countable set $F=\left\{f_{1}, f_{2}, \ldots\right\} C$ $C(X)$ such that $\mu_{n} \rightarrow \mu$ iff $\int f_{k} d \mu_{n} \rightarrow \int f_{k} d \mu$ for $k=1,2, \ldots$. We may assume $\left\|f_{k}\right\|=1$.

Write $F_{k}$ for the set $\left\{f_{1}, \cdots, f_{k}\right\}$. For $\mu \in M(T)$ denote by $U_{k}(\mu)$ the neighborhood

$$
\left\{\nu \in M(T):\left|\int f d \mu-\int f d \nu\right| \leq 2^{-k}, f \in F_{k}\right\} \text {. }
$$


For fixed $k$ there are finitely many CO-measures $\mu_{1}^{k}, \cdots, \mu_{m_{k}}^{k}$ such that $\bigcup_{j=1}^{m_{k}} U_{k}\left(\mu_{j}^{k}\right)$ covers $V$. Since $V$ is connected, we may assume that the $\mu_{j}^{k}$ are enumerated in such a way that $U_{k}\left(\mu_{j}^{k}\right) \cap U_{k}\left(\mu_{j+1}^{k}\right) \cap V \neq \varnothing, j=1, \ldots, m_{k}-1$, and $U_{k}\left(\mu_{m_{k}}^{k}\right) \cap U_{k+1}\left(\mu_{1}^{k+1}\right) \cap V \neq \varnothing, \mu_{j}^{k}$ is generated by some periodic point $x_{j}^{k}$. For simplicity write $U_{n}=U_{k}\left(\mu_{j}^{k}\right), x_{n}=x_{j}^{k}$ and $\mu_{n}=\mu_{j}^{k}$ with $n=m_{1}+m_{2}+\cdots+$ $m_{j-1}+k, 0 \leq k<m_{j}$. Thus $\mu_{n} \in U_{n}$ and $U_{n} \cap U_{n+1} \cap V \neq \varnothing$. We may assume that $x_{n}$ has prime period $p_{n}$.

Now choose a sequence of disjoint strings of integers $A_{n}=\left[a_{n}, b_{n}\right](n=1$, $2, \ldots$ ) such that

(a) the gaps are long enough to allow specification up to $2^{-n}$ along the string $A_{n}$;

(b) the length of $A_{n}$ is much larger than the length of what came before: more precisely, $a_{n} / b_{n} \downarrow{ }^{n}$;

(c) the length of $A_{n}$ is much larger than the length of the gap between $A_{n}$ and $A_{n+1}$. More precisely, $\left(a_{n+1}-b_{n}\right) /\left(b_{n}-a_{n}\right) \downarrow 0$;

(d) $\left(p_{n-1}+p_{n}\right) /\left(b_{n-1}-a_{n-1}\right) \perp 0$.

By (a) and the orbit specification lemma, there is an $x \in X$ such that

$$
d\left(T^{j} x_{0} T^{j} x_{n}\right) \leqslant 2^{-n}, \quad \text { for } j \in A_{n}, n=1,2, \ldots \text {. }
$$

Claim 1. $V \subset V^{T^{l}}(x)$ for $l=1,2, \ldots$.

Proof. Fix $l$ and consider some $\mu \in V$. Choose a sequence $n_{k} \uparrow_{\infty}$ such that $\mu \in U_{n_{k}}$ for $k=1,2, \ldots$. Let $M_{k}$ and $b_{k}$ be integers such that $0 \leq b_{k}<l$ and $M_{k} \cdot l=b_{n_{k}}+b_{k} \cdot$ We shall show that $\mu\left(x, T^{l} ; M_{k}\right) \rightarrow \mu$ for $k \rightarrow \infty$. Fix $f$ $\epsilon F$. If $k$ is large, the expression

$$
\frac{1}{M_{k}} \sum_{j=0}^{M_{k}-1} f\left(T^{j} x\right)
$$

differs very little from

$$
\frac{1}{M_{k}} \sum_{i=0}^{b_{n_{k}}} f\left(T^{i} x\right)
$$

where the dash indicates that one takes the sum only over those $i$ with $i \equiv$ $0(\bmod l)$. This in tum is by (b) very nearly

$$
\frac{l}{b_{n_{k}}-a_{n_{k}}} \sum_{i \in A_{n_{k}}^{\prime}} f\left(T^{i} x\right)
$$

One has by (1) that

$$
\left|\frac{l}{b_{n_{k}}-a_{n_{k}}} \sum_{i \in A_{n_{k}}}^{\prime}\left\{f\left(T^{i} x\right)-f\left(T^{i} x_{n_{k}}\right)\right\}\right| \leq \omega\left(f ; 2^{-n_{k}}\right)
$$


where $\omega(f ; \epsilon)$ is the oscillation of $f$. Using (d),

$$
\left\{\frac{l}{b_{n_{k}}-a_{n_{k}}} \sum_{i \in A_{n_{k}}}^{\prime} f\left(T^{i} x_{n_{k}}\right)-\int f d \mu\left(x_{n_{k}}, T^{l}\right)\right\} \rightarrow 0
$$

for $k \rightarrow \infty$. Since the period $p_{n_{k}}$ of $x_{n_{k}}$ is prime, one has

$$
\int f d \mu\left(x_{n_{k}}, T^{l}\right)=\int f d \mu\left(x_{n_{k}}, T\right)
$$

and this last term tends to $\int f d \mu$. It follows that the expressions in (4), (3) and (2) have $\int f d \mu$ as a limit for $k \rightarrow \infty$, hence $\mu \in V^{T}(x)$.

Claim 2. $V^{T l}(x) \subset V$ for $l=1,2, \ldots$.

Proof. Let $\mu \in V^{T}(x)$ be given. There is a sequence $M_{k} \uparrow_{\infty}$ such that

$$
\mu\left(x, T^{l} ; M_{k}\right) \rightarrow \mu \text { for } k \rightarrow \infty \text {. }
$$

We will construct a sequence $\bar{\mu}_{k} \in V$ such that $\bar{\mu}_{k} \rightarrow \mu_{0}$. Let $b_{k}$ be such that

$$
M_{k} \cdot l=a_{n_{k}}+b_{k} \text { with } 0 \leq b_{k}<a_{n_{k}+1}-a_{n_{k}} \text {. }
$$

One has $\mu_{n_{k}-1} \in U_{n_{k}-1}, \mu_{n_{k}} \in U_{n_{k}}$ and there is a $\bar{\mu}_{k} \in U_{n_{k}-1} \cap U_{n_{k}} \cap V$. By (5) it is enough to show that for all $f \in F$,

$$
\lim _{k \rightarrow \infty}\left\{\frac{1}{M_{k}} \sum_{j=0}^{M_{k}-1} f\left(T^{j l} x\right)-\int f d \mu_{k}\right\}=0 .
$$

Remark first that we may assume that $M_{k} l$ belongs to $A_{n_{k}}$. Indeed, otherwise $M_{k}$ would be, by (6), in the gap between $A_{n_{k}}$ and $A_{n_{k+1}}$. In this case we could replace $M_{k}$ by $M_{k}^{\prime}$, the largest integer $M$ such that $M l$ is still in $A_{n_{k}}$, since by (b) and (c)

$$
\lim _{k \rightarrow \infty}\left\{\frac{1}{M_{k}} \sum_{j=0}^{M_{k}-1} f\left(T^{j l} x\right)-\frac{1}{M_{k}^{\prime}} \sum_{j=0}^{M_{k}^{\prime}-1} f\left(T^{j l} x\right)\right\}=0 .
$$

Now

(8)

$$
\frac{1}{M_{k}} \sum_{j=0}^{M_{k}-1} f\left(T^{j l} x\right)
$$

$$
=\frac{1}{M_{k}}\left(\sum_{i<a_{n_{k}-1}}^{\prime}+\sum_{i \in A_{n_{k}-1}^{\prime}}^{\prime}+\sum_{b_{n_{k}-1}<i \leq a_{n_{k}}}^{\prime}+\sum_{i \leq M_{k} \cdot l ; i \in A_{n_{k}}}^{\prime} f\left(T^{i} x\right)\right) .
$$


Since $M_{k} l \geq a_{n_{k}}$, the first term on the right hand side tends to zero by (b), and the third term tends to zero by (c). The remaining sum differs from

$$
\frac{1}{M_{k}}\left\{\sum_{i \in A} \sum_{n_{k}-1}^{\prime} f\left(T^{i} x_{n_{k}-1}\right)+\sum_{i \in A_{n_{k}} ; i \leq M_{k} l}^{\prime} f\left(T^{i} x_{n_{k}}\right)\right\}
$$

by less than $\omega\left(f ; 2^{-n_{k}+1}\right)+\omega\left(f ; 2^{-n_{k}}\right)$, which tends to zero. Define integers $q$ and $q^{\prime}$ by

$$
\#\left\{i \in A_{n_{k}-1}: i \equiv 0(\bmod l)\right\}=q p_{n_{k}-1}+q^{\prime}, \quad 0 \leq q^{\prime}<p_{n_{k}-1} .
$$

Thus $T^{i} x_{n_{k}-1}$ runs, for $i \in A_{n_{k}-1}$ and $i \equiv 0(\bmod l), q$ times through the entire orbit of $x_{n_{k}-1}$, plus $q^{\prime}$ more steps. Similarly $T^{i} x_{n_{k}}$ runs, for $i \in A_{n_{k}}$, $i \leq M_{k} l$, and $i \equiv 0(\bmod l), r$ times through the entire orbit of $x_{n_{k}}$, plus some $r$ more steps $\left(0 \leq r^{\prime}<p_{n_{k}}\right)$. Clearly

$$
\frac{1}{M_{k}}\left\{\sum_{i \in A}^{\prime} f\left(T^{i} x_{n_{k}-1}\right)-q p_{n_{k}-1} \int f d \mu\left(x_{n_{k}-1}, T^{l}\right)\right\} \leq \frac{q^{\prime}}{M_{k}}
$$

and

$$
\frac{1}{M_{k}}\left\{\sum_{i \in A_{n_{k}} ; i \leq M_{k} l}^{\prime} f\left(T^{i} x_{n_{k}}\right)-r p_{n_{k}} \int f d \mu\left(x_{n_{k}}, T^{l}\right)\right\} \leq \frac{r^{\prime}}{M_{k}}
$$

The right-hand sides of $(10)$ and (11) together are smaller than $\left(p_{n_{k}-1}+p_{n_{k}}\right) / M_{k}$, and tend therefore to 0 by (d).

Denoting by $S_{k}$ the sum in (9), and remarking that $\mu\left(x_{n_{k}-1}, T^{l}\right)=\mu\left(x_{n_{k}-1}, T\right)$ $=\mu_{n_{k}-1}$ and $\mu\left(x_{n_{k}}, T\right)=\mu_{n_{k}}$, one obcains

$$
\lim _{k \rightarrow \infty}\left\{S_{k}-\frac{1}{M_{k}}\left(q p_{n_{k}-1} \int f d \mu_{x_{n_{k}-1}}+r p_{n_{k}} \int f d \mu_{x_{n_{k}}}\right)\right\}=0
$$

Let $a=\left(q \cdot p_{n_{k}-1}\right) /\left(q \cdot p_{n_{k}-1}+r \cdot p_{n_{k}}\right)$. By simple estimates using (b) and (c), one obtains

$$
\lim _{k \rightarrow \infty}\left\{s_{k}-\left(\alpha \int f d \mu_{n_{k}-1}+(1-\alpha) \int f d \mu_{n_{k}}\right)\right\}=0
$$

Now $f$ is in some $F_{k}$. Since $\bar{\mu}_{k} \in U_{n_{k}-1} \cap U_{n_{k}}$, one has 


$$
\lim _{k \rightarrow \infty}\left(\int f d \mu_{x_{n_{k}-1}}-f d \bar{\mu}_{k}\right)=0, \quad \lim _{k \rightarrow \infty}\left(\int f d \mu_{x_{n_{k}}}-\int f d \bar{\mu}_{k}\right)=0
$$

and hence by (13)

$$
\lim _{k \rightarrow \infty}\left\{S_{k}-\int f d \bar{\mu}_{k}\right\}=0 .
$$

By tracing back through equations (14), (9), and (8), one obtains (7), and hence $\mu \in V$.

For $X=[0,1)$ and $T=T_{s}: x \rightarrow x s(\bmod 1)$ one obtains a theorem of Colebrook [6].

Corollary. Let $(X, T)$ be a system with the specification property and $\mu \epsilon$ $M(T)$. Then there exists an $x \in X$ which is a generic point for $\mu$ with respect to $T, T^{2}, T^{3}, \ldots$ etc.

4. Quasiregular points. A point $q \in X$ is said to be quasiregular with respect to the continuous map $T$ from $X$ onto itself if $q$ is generic for some $T$. invariant measure on $X$, i.e. if $V^{T}(q)$ consists of exactly one point in $M(T)$. Let $Q(T)$ denote the set of quasiregular points. It is well known that $\mu(Q(T))=1$ for all $\mu \in M(T)$ (see [12]). Thus $Q(T)$ is large, measure theoretically. From the topological point of view, however, it is often small. This happens, in particular, if $(X, T) \in$ SPEC.

Theorem 5. If $(X, T)$ is a nontrivial system with the specification property, then $Q(T)$ is a dense set of first category in $X$, containing c points.

Proof. That $Q(T)$ is dense follows trivially from Theorem 1. That $Q(T)$ has $c$ points follows from Theorem 4 and the fact that $M(T)$ has $c$ points. There clearly exist points in $X$ whose orbits are dense and which do not belong to $Q(T)$. (Take for example an $x$ with $V^{T}(x)=M(T)$.) It follows from [7, Theorem 3] that $Q(T)$ is of first category in $X$.

As a special case of this theorem one has that the set of normal points is of first category in $[0,1)$. This is known at least since [13]. In [0] Furstenberg proved that $Q(T) \neq X$ if $T$ is an ergodic automorphism of the twotorus $X$. Theorem 5 sharpens this result. In [22] it is shown that if $S$ and $T$ are distinct ergodic group automorphisms of the twotorus, then $Q(T) \neq Q(S)$. In the proof one uses the following:

Theorem 6. Let $(X, T)$ be a system with the specification property. Then $Q\left(T^{k}\right) \neq Q\left(T^{l}\right)$ if $k \neq l$.

Proof. (i) If $k=0$ and $l \neq 0$ the theorem follows from Theorem 5 and Proposition 1(a): $Q\left(T^{l}\right) \not X$. 
(ii) In order to show that $Q\left(T^{k}\right) \neq Q\left(T^{l}\right)$ for $k, l>0, k \neq l$, we prove the following statement: Let $(X, T) \in \mathrm{SPEC}$, and let $S: X \rightarrow X$ be a continuous map such that $T^{k}=S^{l}$ for some $k, l>0$, but $T \neq S$. Then $Q(T) \neq Q(S)$.

Remark first that by Proposition $1(a),(X, S) \in$ SPEC. Since $S$ and $T$ are different, there exists an $x$ such that either the S-orbit of $x$ is distinct from the S-orbit of $T x$, or the T-orbit of $x$ is distinct from the T-orbit of Sx. Let us assume the first case. By the specification property we may assume that $x$ is a periodic point of $S$.

With help of the orbit specification lemma, applied to $S$, one can choose a sequence of disjoint strings of integers $A_{n}=\left[a_{n}, b_{n}\right], n>0$, such that conditions (a), (b) and (c) in the proof of Theorem 4 are satisfied.

Now define $x_{n} \in X$ by $x_{n}=x$ if $n$ is even, $x_{n}=T x$ if $n$ is odd (recall that $T x \neq x)$. Let $z \in X$ be specified such that, for $j \in A_{n}, d\left(S^{j} z, S^{j} x_{n}\right) \leq 2^{-n}$.

Such a $z$ exists by (a). Thus $S^{j} z$ runs along the S-orbit of $x$ for $j \in A_{1}$; it runs along the S-orbit of $T x$ for $j \in A_{2}$; again along the S-orbit of $x$ for $j \in A_{3}$; etc.

Claim 1. $z \notin Q(S)$. Indeed, since the S-orbits of $x$ and $T x$ are two distinct finite sets, there exists an $f \in C(X)$ such that

$$
\lim _{N \rightarrow \infty} \frac{1}{N} \sum_{j=0}^{N-1} f\left(S^{j} x\right) \neq \lim _{N \rightarrow \infty} \frac{1}{N} \sum_{j=0}^{N-1} f\left(S^{j} T x\right) .
$$

Now by (b)

$$
\lim _{n \rightarrow \infty} \frac{1}{b_{n}} \sum_{j=0}^{b_{n}-1} f\left(S^{j} z\right)=\lim _{n \rightarrow \infty} \frac{1}{b_{n}-a_{n}} \sum_{j=a_{n}}^{b_{n}-1} f\left(S^{j} z\right)
$$

and this last expression is

$$
\begin{aligned}
& \lim _{N \rightarrow \infty} \frac{1}{N} \sum_{j=0}^{N-1} f\left(S^{j} x\right) \text { for } x \text { even, } \\
& \lim _{N \rightarrow \infty} \frac{1}{N} \sum_{j=0}^{N-1} f\left(S^{j} T x\right) \text { for } x \text { odd. }
\end{aligned}
$$

(It is easy to see that the limits in (2) exist.)

Thus $\lim _{N \rightarrow \infty} N^{-1} \Sigma_{j=0}^{N-1} f\left(S^{j} z\right)$ does not exist, $z \notin Q(S)$.

Claim 2. $z \in Q(T)$. Indeed, for $N>0$ let $n$ be such that $b_{n} \leq N<b_{n+1}$ 。 Then for $f \in C(X)$ and $N$ large, one has 


$$
\frac{1}{N} \sum_{j=0}^{N-1} f\left(T^{j} z\right) \sim \frac{1}{N}\left\{\sum_{j \in A_{n}} f\left(T^{j} z\right)+\sum_{j \in A_{n+1} ; j<N} f\left(T^{j} z\right)\right\}
$$

Indeed, one can forget about the terms $f\left(T^{j} z\right)$ with $j<a_{n}$ by (b), and about those with $j$ in the gap between $A_{n}$ and $A_{n+1}$, by (c). The righthand side of (3) is almost equal to

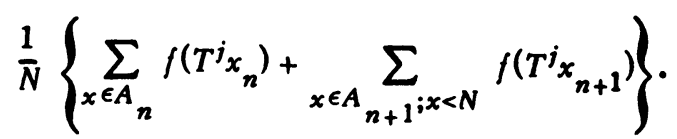

which in turn is equal to

$$
\begin{aligned}
& \frac{1}{N}\left\{\sum_{j \in A} f\left(T^{j} x\right)+\sum_{j \in A_{n+1} ; j<N} f\left(T^{j} T x\right)\right\} \text { for } j \text { even, } \\
& \frac{1}{N}\left\{\sum_{j \in A} f\left(T^{j} T x\right)+\sum_{j \in A_{n+1} ; j<N} f\left(T^{j} x\right)\right\} \text { for } j \text { odd. }
\end{aligned}
$$

Now $x$ is a periodic point for $S$ and thus, since $S^{l}=T^{k}$, it is periodic for $T$, with period $p$, say. If $N$ is large enough, card $\left(A_{n}\right)$ is much larger than $p$. It is easy to check that for $N \rightarrow \infty$, the expressions (5) and $\left(5^{\prime}\right)$ tend to

$$
\frac{1}{p} \sum_{j=0}^{p-1} f\left(T^{j} x\right)
$$

Hence

$$
\lim _{N \rightarrow \infty} \frac{1}{N} \sum_{j=0}^{N-1} f\left(T^{j} z\right)
$$

exists, $z \in Q(T)$.

(iii) If $T$ is a homeomorphism and $k, l>0$, then it is easy to construct a $z \in Q\left(T^{k}\right)$ with $z \notin Q\left(T^{-l}\right)$. Indeed, choose $x \in Q\left(T^{k}\right)$ and $y \in X \backslash Q\left(T^{-l}\right)$. Let $A_{n}, n \in Z$, be a sequence of increasing disjoint strings of integers, with gaps large enough such that, by the orbit specification lemma, there exists a $z$ with

$$
\begin{array}{ll}
d\left(T^{j} x, T^{j} z\right)<2^{-n} & \text { for } j \in A_{n^{\prime}} n>0, \\
d\left(T^{j} y, T^{j} z\right)<2^{-n} & \text { for } j \in A_{n^{\prime}} n<0 .
\end{array}
$$


One can choose the strings so long and the gaps so short that $\bigcup_{n>0} A_{n}$ (resp. $\left.\cup_{n<0} A_{n}\right)$ is of density 1 in $Z^{+}$(resp. $-Z^{+}$). Then $z \in Q\left(T^{k}\right)$ and $z \notin Q\left(T^{-l}\right)$.

\section{REFERENCES}

1. R. Bowen, Topological entropy and axiom A, Proc. Sympos. Pure Math., vol. 14, Amer. Math. Soc., Providence, R. 1., 1970, pp. 23-41. MR 41 \#7066.

2. - Periodic points and measures for Axiom A diffeomorphisms, Trans. Amer. Math. Soc. 154 (1971), 377-397. MR 43 \#8084.

3. —_ Entropy for group endomorphisms and homogeneous spaces, Trans. Amer. Math. Soc. 153 (1971), 401-414. MR 43 \#469.

4. - Periodic orbits for hyperbolic flows, Amer. J. Math. 94 (1972), 1-30. MR $45 \# 7749$.

5. - Some systems with unique equilibrium states. (to appear).

6. C. Colebrook, The Hausdorff dimension of certain sets of nonnormal numbers, Michigan Math. J. 17 (1970), 103-116. MR 41 \#5321.

7. Y. Dowker, The mean and transitive points of homeomorphisms, Ann. of Math. (2) 58 (1953), 123-133. MR 14, 1003.

8. H. Eggleston, The fractional dimension of a set defined by decimal properties, Quart. J. Math. Oxford Ser. 20 (1949), 31-36. MR 11, 88.

9. H. Furstenberg, Strict ergodicity and transformations on the torus, Amer. J. Math. 83 (1961), 573-601. MR $24 \#$ \#3263.

10. - Disjointness in ergodic theory, minimal sets, and a problem in Diophantine approximation, Math. Systems Theory 1 (1967), 1-49. MR 35 \#4369.

11. M. Hirsch, Expanding maps and transformation groups, Global Analysis, Proc. Sympos. Pure Math., vol. 14, Amer. Math. Soc., Providence, R. I., 1970, pp. 125-131. MR $45 \# 7750$.

12. J. Oxtoby, Ergodic sets, Bull. Amer. Math. Soc. 58 (1952), 116-136. MR 13, 850.

13. J. Oxtoby and S. Ulam, Measure preserving homeomorphisms and metrical transitivity, Ann. of Math. (2) 42 (1941), 874-920. MR 3, 211.

14. K. R. Parthasarathy, A note on mixing processes, Sankhyā, Ser. A 24 (1962), 331-332. MR $29 \# 6535$.

15. - Probability measures on metric spaces, Probability and Math. Statistics, no. 3, Academic Press, New York, 1967. MR 37 \#2271.

16. D. Ruelle, Statistical mechanics on a compact set with $Z^{\nu}$ action satisfying expansiveness and specification (to appear).

17. M. Shub, Endomorphisms of compact differentiable manifolds, Amer. J. Math. 91 (1969), 175-199. MR 39 \#2169.

18. K. Sigmund, Generic properties of invariant measures for Axiom A diffeomorphisms, Invent. Math. 11 (1970), 99-109.

19. _- Ergodic averages for Axiom A diffeomorphisms, Z. Wahrscheinlichkeitstheorie Verw. Gebiete 20 (1971), 319-324.

20. —_, Mixing measures for Axiom A diffeomorphisms, Proc. Amer. Math. Soc. 36 (1972), 497-504.

21. On the space of invariant measures for hyperbolic flows, Amer. J. Math. 94 (1972), 31-37. 
22. K. Sigmund, On normal and quasiregular points for automorphisms on the torus, Math. System Theory (to appear).

23. B. Volkmann, Über Hausdorffsche Dimensionen von Mengen, die durch Ziffermeigenschaften charakterisiert sind. VI, Math. Z. 68 (1958), 439-449, MR 20 \#7008.

24. B. Weiss, Topological transitivity and ergodic measures, Math. System Theory 5 (1971), 71-75. MR 45 \#5987.

25. —_ Subshifts of finite type and sofic systems, Monatsh. Math. 77 (1973), 462-474.

MATHEMATISCHES INSTITUT DER UNIVERSITÄT, A-1090 WIEN, STRUDLHOFGASSE 4. AUSTRIA

Current address: Institut fữ Mathematische Statistik, Universitåt Göttingen, Lotzestrasse 13 , Göttingen, Germany 